\title{
Equality of consequence relations in finite-valued logical matrices
}

\author{
LEONID YU. DEVYATKIN
}

\begin{abstract}
In this paper the procedure is presented that allows to determine in finite number of steps if consequence relations in two finite-valued logical matrices for propostional language $L$ are equal.

Keywords: product of logical matrices, consequence relation, equality of matrices
\end{abstract}

In his paper 'A test for the equality of truth-tables' [2], J. Kalicki has described a general method for testing the equality of the classes of tautologies in different finite-valued matrices. Below I present a generalization of Kalicki's method which allows to test whether the consequence relations in two finite-valued logical matrices are equal.

First, the question of equality of consequence relations in two arbitrary matrices will be reduced to the question of the properties of a single matrix. This matrix will be obtained from initial matrices via the operation of product, but it will have four classes of truthvalues instead of the standard two (designated and non-designated). On the basis of these four classes I will define several consequence relations. The properties that these relations display in the product matrix will define if two initial matrices are equal in terms of consequence relation. Then I will show that it is sufficient to consider a finite set of formulas to investigate the properties in question, and that therefore a finite number of steps is required to determine if consequence relations are equal in two finite-valued matrices.

Let us begin with some necessary definitions. 
Definition 1. A logical matrix is a structure $\mathfrak{M}=\langle V, F, D>$, where $V$ is the set of truth-values, $F$ is a set of functions on $V$ called basic functions, and $D$ is a designated subset of $V$.

In this paper we will only consider the logical matrices where $V$ is finite.

If for any $n$ it is true that $\mathfrak{M}$ contains as much $n$-ary elements of $F$ as there are $n$-ary connectives in some propositional language $L$, $\mathfrak{M}$ is a logical matrix for $L$. In that case we can establish a one-toone correspondence between the elements of $F$ and the connectives of $L$, and define a valuation of a formula in $\mathfrak{M}$.

Definition 2. A valuation $v$ of formula $A$ in $\mathfrak{M}$ is a homomorphism of $L$ in $\langle V, F\rangle$ such that

1. if $A$ is a propositional variable, then $v(A) \in V$;

2. if $A_{1}, A_{2}, \cdots, A_{n}$ are formulas, and $\mathbb{C}$ is an $n$-ary connective of $L$, then $v\left(\mathbb{C}\left(A_{1}, A_{2}, \cdots, A_{n}\right)\right)=f^{n}\left(v\left(A_{1}\right), v\left(A_{2}\right), \cdots, v\left(A_{n}\right)\right)$, where $f^{n}$ is a function from $F$ corresponding to $\mathbb{C}$.

The definition of consequence relation in $\mathfrak{M}$ is a standard one.

Definition 3. $\Gamma \vDash(\mathfrak{M}) B$ iff there is no valuation $v$ in $\mathfrak{M}$, such that $v[\Gamma] \subseteq D(\mathfrak{M})$ (i.e. every formula from $\Gamma$ assumes a truth-value designated in $\mathfrak{M})$, and $v(A) \notin D(\mathfrak{M})$.

Let us denote as $C(\mathfrak{M})$ a set of ordered pairs $\langle\Gamma, B\rangle$, such that $\Gamma$ is a set of formulas, $B$ is a formula, and $\Gamma \vDash(\mathfrak{M}) B$. Now we will define the equality of consequence relations in two arbitary matrices for $L$.

Definition 4. Let $\mathfrak{A}$ and $\mathfrak{B}$ be the matrices for $L$. The consequence relations in $\mathfrak{A}$ and $\mathfrak{B}$ are equal iff $C(\mathfrak{A})=C(\mathfrak{B})$.

Now we will make the transition from two matrices to one by applying the product operation. If $\mathfrak{A}$ and $\mathfrak{B}$ are the matrices for $L$, a one-to-one correspondence between the elemnts of their sets of basic functions can be established. This allows us to give the following definition.

Definition 5. A product of matrices $\mathfrak{A}$ and $\mathfrak{B}$ is a matrix $\mathfrak{C}=$ $\mathfrak{A} \otimes \mathfrak{B}$, such that 
- $V(\mathfrak{C})$ is a Cartesian product of $V(\mathfrak{A})$ and $V(\mathfrak{B})$;

- for each pair pair of mutually corresponding $k$-ary basic functions $f^{k}\left(x_{1}, x_{2}, \cdots, x_{k}\right)$ from $\mathfrak{A}$ and $g^{k}\left(y_{1}, y_{2}, \cdots, y_{k}\right)$ from $\mathfrak{B}$ there is one and only one basic operation $h^{k}$ from $\mathfrak{C}$, and $h^{k}\left(<x_{1}, y_{1}>,<x_{2}, y_{2}>, \cdots,<x_{k}, y_{k}>\right)=$ $<f^{k}\left(x_{1}, x_{2}, \cdots, x_{k}\right), g^{k}\left(y_{1}, y_{2}, \cdots, y_{k}\right)>$.

This is a standard product operation. However, the truth-values in $\mathfrak{C}$ will be divided into four classes ${ }^{1}$ :

- $<x_{i}, y_{j}>\in \omega(\mathfrak{C})$ iff $x_{i} \in D(\mathfrak{A})$ and $y_{j} \in D(\mathfrak{B})$;

- $<x_{i}, y_{j}>\in \xi(\mathfrak{C})$ iff $x_{i} \in D(\mathfrak{A})$ and $y_{j} \notin D(\mathfrak{B})$;

- $<x_{i}, y_{j}>\in \xi^{\prime}(\mathfrak{C})$ iff $x_{i} \notin D(\mathfrak{A})$ and $y_{j} \in D(\mathfrak{B})$;

- $<x_{i}, y_{j}>\in \phi(\mathfrak{C})$ iff $x_{i} \notin D(\mathfrak{A})$ and $y_{j} \notin D(\mathfrak{B})$.

I will now consider two definitions of consequense relation based on these four classes, $\vDash_{\cup}$ and $\vDash_{n}$.

Definition 6. $\Gamma \vDash_{\cup}(\mathfrak{C}) B$ iff there is no valuation $w$ in $\mathfrak{C}$, such that $w[\Gamma] \subseteq \omega(\mathfrak{C})$, and $w(A) \in \phi(\mathfrak{C})$.

Lemma 1. $\Gamma \vDash_{\cup}(\mathfrak{C}) B$ iff $\Gamma \vDash(\mathfrak{A}) B$ or $\Gamma \vDash(\mathfrak{B}) B$.

Proof. (i) Let $\Gamma \vDash_{\cup}(\mathfrak{C}) B$, and $\Gamma \not \models(\mathfrak{A}) B$, and $\Gamma \not \models(\mathfrak{B}) B$. Then there exists a valuation $v^{*}$ in $\mathfrak{A}$, such that $v^{*}[\Gamma] \subseteq D(\mathfrak{A})$ and $v^{*}(A) \notin$ $D(\mathfrak{A})$, and there exists a valuation $u^{*}$ in $\mathfrak{B}$, such that $u^{*}[\Gamma] \subseteq D(\mathfrak{B})$ and $u^{*}(A) \notin D(\mathfrak{B})$. For every $v$ and $u$ there is a mapping $w$ of the propositional variables of $L$ on $V(\mathfrak{A}) \times V(\mathfrak{B})$, such that $w\left(p_{k}\right)=<$ $v\left(p_{k}\right), u\left(p_{k}\right)>$, where $p_{k}$ is a propositional variable. Obviously, every such $w$ is a valuation in $\mathfrak{C}$. By definition of $\mathfrak{C}, w^{*}$ obtained from $v^{*}$ and $u^{*}$ is such a valuation that $w^{*}[\Gamma] \subseteq \omega(\mathfrak{C})$, and $w^{*}(A) \in \phi(\mathfrak{C})$. That contradicts our assumption.

(ii) Let $\Gamma \not \nvdash_{\cup}(\mathfrak{C}) B$, and $\Gamma \vDash(\mathfrak{A}) B$ or $\Gamma \vDash(\mathfrak{B}) B$. Then there is a valuation $w^{*}$ in $\mathfrak{C}$, such that $w^{*}[\Gamma] \subseteq \omega(\mathfrak{C})$, and $w^{*}(A) \in \phi(\mathfrak{C})$. For

\footnotetext{
${ }^{1}$ This is essentially a distribution introduced by Kalicki [2], but he only needed three classes, so elements of $\xi(\mathfrak{C})$ and $\xi^{\prime}(\mathfrak{C})$ were assigned to the same class.
} 
every valuation $w$ in $\mathfrak{C}$ there is the following valuation $v$ in $\mathfrak{A}$ : if $w\left(p_{k}\right)=<x_{i}, y_{j}>$, then $v\left(p_{k}\right)=x_{i}$. By definition of $\mathfrak{C}, v^{*}$ obtained this way from $w^{*}$ is such a valuation in $\mathfrak{A}$ that $v^{*}[\Gamma] \subseteq D(\mathfrak{A})$ and $v^{*}(A) \notin D(\mathfrak{A})$. The reasoning for valuation $u^{*}$ in $\mathfrak{B}$ is analogous, and leads to the contradiction.

Definition 7. $\Gamma \vDash_{\cap}(\mathfrak{C}) B$ iff all three of the following conditions are fulfilled:

- there is no valuation $w$ in $\mathfrak{C}$, such that $w[\Gamma] \subseteq \omega(\mathfrak{C})$, and $w(A) \notin \omega(\mathfrak{C})$;

- there is no valuation $w$ in $\mathfrak{C}$, such that $w[\Gamma] \subseteq \omega(\mathfrak{C}) \cup \xi(\mathfrak{C})$, and $w(A) \notin \omega(\mathfrak{C}) \cup \xi(\mathfrak{C})$;

- there is no valuation $w$ in $\mathfrak{C}$, such that $w[\Gamma] \subseteq \omega(\mathfrak{C}) \cup \xi^{\prime}(\mathfrak{C})$, and $w(A) \notin \omega(\mathfrak{C}) \cup \xi^{\prime}(\mathfrak{C})$.

Lemma 2. $\Gamma \vDash_{\cap}(\mathfrak{C}) B$ iff $\Gamma \vDash(\mathfrak{A}) B$ and $\Gamma \vDash(\mathfrak{B}) B$.

Proof. (i) Let $\Gamma \vDash_{\cap}(\mathfrak{C}) B$, and $\Gamma \not \models(\mathfrak{A}) B$, and $\Gamma \not \models(\mathfrak{B}) B$. The reasoning is analogous to the one in Lemma 1.

(ii) Let $\Gamma \vDash_{\cap}(\mathfrak{C}) B$, and either $\Gamma \not \models(\mathfrak{A}) B$ or $\Gamma \not \models(\mathfrak{B}) B$. Suppose $\Gamma \not \models(\mathfrak{A}) B$ and $\Gamma \vDash(\mathfrak{B}) B$. Then there is a valuation $v^{*}$ in $\mathfrak{A}$, such that $v^{*}[\Gamma] \subseteq D(\mathfrak{A})$ and $v^{*}(A) \notin D(\mathfrak{A})$. Now we have to consider two possibilities.

(ii.1) There is a valuation $u^{*}$ in $\mathfrak{B}$, such that $u^{*}[\Gamma] \subseteq D(\mathfrak{B})$ and $u^{*}(A) \in D(\mathfrak{B})$. In this case, from $v^{*}$ and $u^{*}$ we can obtain a corresponding valuation $w^{*}$ in $\mathfrak{C}$ (see Lemma 1 ), such that $w^{*}[\Gamma] \subseteq$ $\omega(\mathfrak{C})$, and $w^{*}(A) \in \xi^{\prime}(\mathfrak{C})$. But then $\Gamma \not \nvdash_{\cap}(\mathfrak{C}) B$, which contradicts our assumption.

(ii.2) For every valuation $u$ in $\mathfrak{B}, u[\Gamma] \notin D(\mathfrak{B})$. Let $u^{\prime}$ be such a valuation that $u^{\prime}[\Gamma] \notin D(\mathfrak{B})$, and $u^{\prime}(A) \notin D(\mathfrak{B})$. The corresponding valuation $w^{\prime}$ in $\mathfrak{C}$ obtained from $v^{*}$ and $u^{\prime}$ in the same way as in Lemma 1 will be such that $w^{\prime}[\Gamma] \subseteq \xi(\mathfrak{C})$, and $w^{\prime}(A) \in \phi(\mathfrak{C})$. Let $u^{\prime \prime}$ be such a valuation that $u^{\prime \prime}[\Gamma] \notin D(\mathfrak{B})$, and $u^{\prime \prime}(A) \in D(\mathfrak{B})$. The corresponding valuation $w^{\prime \prime}$ in $\mathfrak{C}$ obtained from $v^{*}$ and $u^{\prime \prime}$ will be such that $w^{\prime \prime}[\Gamma] \subseteq \xi(\mathfrak{C})$, and $w^{\prime \prime}(A) \in \xi^{\prime}(\mathfrak{C})$. Both cases lead us to the contradiction with the assumption that $\Gamma \vDash_{\cap}(\mathfrak{C}) B$. 
The reasoning for $\Gamma \vDash(\mathfrak{A}) B$ and $\Gamma \not \models(\mathfrak{B}) B$ is analogous.

(iii) Let $\Gamma \not \not_{\cap}(\mathfrak{C}) B$, and $\Gamma \vDash(\mathfrak{A}) B$, and $\Gamma \vDash(\mathfrak{B}) B$. If $\Gamma \not \not_{\cap}(\mathfrak{C}) B$, three cases are possible:

(iii.1) There is a valuation $w$ in $\mathfrak{C}$, such that $w[\Gamma] \subseteq \omega(\mathfrak{C})$, and $w(A) \notin \omega(\mathfrak{C}) ;$

(iii.2) There is a valuation $w$ in $\mathfrak{C}$, such that $w[\Gamma] \subseteq \omega(\mathfrak{C}) \cup \xi(\mathfrak{C})$, and $w(A) \notin \omega(\mathfrak{C}) \cup \xi(\mathfrak{C})$;

(iii.3) There is a valuation $w$ in $\mathfrak{C}$, such that $w[\Gamma] \subseteq \omega(\mathfrak{C}) \cup \xi^{\prime}(\mathfrak{C})$, and $w(A) \notin \omega(\mathfrak{C}) \cup \xi^{\prime}(\mathfrak{C})$.

The reasining for all three cases is the same. We obtain from $w$ the corresponing valuations $v$ in $\mathfrak{A}$ and $u$ in $\mathfrak{B}$ in the same way as we did in Lemma 1. Due to the properties of $w$ described in (iii.1)-(iii.3), either $v$, or $u$, or both of them will be such that they will lead to the contradiction with the assumption that $\Gamma \vDash(\mathfrak{A}) B$ and $\Gamma \vDash(\mathfrak{B}) B$.

From Lemma 1 we have that $C\left(\mathfrak{C}, \vDash_{\cup}\right)=C(\mathfrak{A}) \cup C(\mathfrak{B})$. From Lemma 2 we have that $C\left(\mathfrak{C}, \vDash_{\cap}\right)=C(\mathfrak{A}) \cap C(\mathfrak{B})$. Also, we have that $C(\mathfrak{A})=C(\mathfrak{B})$ iff $C(\mathfrak{A}) \cup C(\mathfrak{B})=C(\mathfrak{A}) \cap C(\mathfrak{B})$. Therefore, $C(\mathfrak{A})=C(\mathfrak{B})$ iff $C\left(\mathfrak{C}, \vDash_{\cup}\right)=C\left(\mathfrak{C}, \vDash_{\cap}\right)$.

Now let us consider another consequence relation.

Definition 8. $\Gamma \vDash^{*}(\mathfrak{C}) B$ iff either

- there is no valuation $w$ in $\mathfrak{C}$, such that $w[\Gamma] \subseteq \omega(\mathfrak{C})$, and $w(A) \notin \omega(\mathfrak{C})$,

- and there is no valuation $w$ in $\mathfrak{C}$, such that $w[\Gamma] \subseteq \omega(\mathfrak{C}) \cup \xi(\mathfrak{C})$, and $w(A) \notin \omega(\mathfrak{C}) \cup \xi(\mathfrak{C})$,

- and there is no valuation $w$ in $\mathfrak{C}$, such that $w[\Gamma] \subseteq \omega(\mathfrak{C}) \cup \xi^{\prime}(\mathfrak{C})$, and $w(A) \notin \omega(\mathfrak{C}) \cup \xi^{\prime}(\mathfrak{C})$,

- or there is a valuation $w$ in $\mathfrak{C}$, such that $w[\Gamma] \subseteq \omega(\mathfrak{C})$, and $w(A) \in \phi(\mathfrak{C})$.

Lemma 3. $C\left(\mathfrak{C}, \vDash_{\cup}\right)=C\left(\mathfrak{C}, \vDash_{\cap}\right)$ iff $\Gamma \vDash^{*}(\mathfrak{C}) B$ for each set of formulas $\Gamma$ and each formula $B$. 
Proof. If $C\left(\mathfrak{C}, \vDash_{\cup}\right)=C\left(\mathfrak{C}, \vDash_{\cap}\right)$, for each $\Gamma$ and $B$ it is true that either $\Gamma \vDash_{\cap}(\mathfrak{C}) B$ or $\Gamma \nvdash_{\cup}(\mathfrak{C}) B$. Both cases lead to $\Gamma \vDash^{*}(\mathfrak{C}) B$. Now let us assume that $\Gamma \vDash^{*}(\mathfrak{C}) B$ for some arbitrary $\Gamma$ and $B$. Then (i) for every evaluation $w$ in $\mathfrak{C}$, if $w[\Gamma] \subseteq \omega(\mathfrak{C})$ then $w(A) \in \omega(\mathfrak{C})$, if $w[\Gamma] \subseteq \omega(\mathfrak{C}) \cup \xi^{\prime}(\mathfrak{C})$, then $w(A) \in \omega(\mathfrak{C}) \cup \xi^{\prime}(\mathfrak{C})$, if $w[\Gamma] \subseteq \omega(\mathfrak{C}) \cap \xi^{\prime}(\mathfrak{C})$, then $w(A) \in \omega(\mathfrak{C}) \cap \xi^{\prime}(\mathfrak{C})$, or (ii) there is at least one valuation in $\mathfrak{C}$, such that all formulas from $\Gamma$ assume a truth value from $\omega(\mathfrak{C})$, and $B$ assumes a value from $\phi(\mathfrak{C})$. In the first case $\Gamma \vDash_{\cap}(\mathfrak{C}) B$. In the second case $\Gamma \nvdash_{\cup}(\mathfrak{C}) B$. Therefore $C\left(\mathfrak{C}, \vDash_{\cup}\right)=C\left(\mathfrak{C}, \vDash_{\cap}\right)$.

Below, the number of formulas that need to be considered will be narrowed down to a finite set. I will use the method proposed by J. Kalicki in [1] with necessary modifications.

Lemma 4. For each matrix $\mathfrak{C}_{m}$, where $m$ is the number of the elements of $V(\mathfrak{C})$, the following is true: if for each pair $\Gamma$ and $B$ that contains $i \leq m$ different variables $\Gamma \vDash^{*}\left(\mathfrak{C}_{m}\right) B$, then for each pair $\Delta$ and $E$ that contains $m+t(t=0,1, \cdots)$ different variables $\Delta \vDash^{*}\left(\mathfrak{C}_{m}\right) E$.

Proof. Let us use the induction by $t$. For $t=0$ it is obvious that for each $\Gamma$ and $B$ that contains $i \leq m$ different variables $\Gamma \vDash^{*}\left(\mathfrak{C}_{m}\right) B$, then for each pair $\Delta$ and $E$ that contains $m$ different variables $\Delta \vDash^{*}\left(\mathfrak{C}_{m}\right) E$.

Let us assume that the theorem is true for $t \leq k$ and prove it for $t=k+1$. Let there exist $\Delta$ and $E$ that contain $m+k+1$ different variables, and $\Delta \not^{*}\left(\mathfrak{C}_{m}\right) E$. Then there exists a valuation $w_{0}$ in $\mathfrak{C}_{m}$ that maps the variables $p_{1}, p_{2}, \cdots, p_{m+k+1}$ on values $x_{1}, x_{2}, \cdots, x_{m+k+1}$ respectively, such that either (i) $w_{0}[\Delta] \subseteq \omega(\mathfrak{C})$, and $w_{0}(E) \notin \omega(\mathfrak{C})$, or (ii) $w_{0}[\Delta] \subseteq \omega(\mathfrak{C}) \cup \xi(\mathfrak{C})$, and $w(E) \notin$ $\omega(\mathfrak{C}) \cup \xi(\mathfrak{C})$, or $\left(\right.$ iii) $w_{0}[\Delta] \subseteq \omega(\mathfrak{C}) \cup \xi^{\prime}(\mathfrak{C})$, and $w(E) \notin \omega(\mathfrak{C}) \cup \xi^{\prime}(\mathfrak{C})$.

Let us consider (i). Due to the fact that in $\mathfrak{C}_{m}$ there is $m$ different truth-values in total, there will be at least two $i_{1} \neq i_{2}$ among $i=1,2, \cdots, m+k+1$, such that $x_{i_{1}}=x_{i_{2}}$. Now let us consider $\Delta^{\prime}$ and $E^{\prime}$, obtained from $\Delta$ and $D$ by replacement of all instances of $p_{i_{2}}$ with $p_{i_{1}}$. It is clear that $w_{0}\left[\Delta^{\prime}\right] \subseteq \omega\left(\mathfrak{C}_{m}\right)$ and $w_{0}\left(E^{\prime}\right) \notin \omega\left(\mathfrak{C}_{m}\right)$. Because $\Delta^{\prime}$ and $E^{\prime}$ contain $m+k$ different variables, according to the inductive assumption, $\Delta^{\prime} \vDash^{*}\left(\mathfrak{C}_{m}\right) E^{\prime}$. Therefore, there exists a valuation $w^{*}$ in 
$\mathfrak{C}_{m}$, which maps the variables $p_{1}, p_{2}, \cdots, p_{i_{2-1}}, p_{i_{2+1}}, \cdots, p_{m+k+1}$ on the values $y_{1}, y_{2}, \cdots, y_{i_{2-1}}, y_{i_{2+1}}, \cdots, y_{m+k+1}$ respectively, such that $w^{*}\left[\Delta^{\prime}\right] \subseteq \omega\left(\mathfrak{C}_{m}\right)$ and $w^{*}\left(E^{\prime}\right) \in \phi\left(\mathfrak{C}_{m}\right)$. In this case we can construct a valuation $w^{* *}$, which maps the variables $p_{1}, p_{2}, \cdots, p_{m+k+1}$ on the values $y_{1}, y_{2}, \cdots, y_{i_{2-1}}, y_{i_{1}}, y_{i_{2+1}}, \cdots, y_{m+k+1}$ respectively. It is clear that $w^{* *}[\Delta] \subseteq \omega\left(\mathfrak{C}_{m}\right)$ and $w^{* *}(E) \in \phi\left(\mathfrak{C}_{m}\right)$. But then $\Delta \vDash^{*}\left(\mathfrak{C}_{m}\right) E$, which contradicts our assumption.

The reasoning for (ii) and (iii) is analogous.

For $m$ different variables there is $k=m^{m}$ different valuations $v_{1}, v_{2}, \cdots, v_{k}$ in $\mathfrak{C}_{m}$. We can assign to each variable $p_{i}(1 \leq i \leq$ m) a unique value-sequence $\left|p_{i}\right|=<x_{1}, x_{2}, \cdots, x_{k}>$, where $x_{l}=$ $v_{l}\left(p_{i}\right)(1 \leq l \leq k)$.

Now let us construct the following sequence of the classes of formulas:

- The elements of $C L_{0}$ are the variables $p_{1}, p_{2}, \cdots, p_{m}$ exclusively;

- to a class $C L_{t+1}$ belong all formulas that can be constructed by means of one connective, an element of class $C L_{t}$, and (if needed) elements of $C L_{n \leq t}$.

For each formula $B$ from $C L_{n}$ we can calculate the corresponding value-sequence $|B|=<y_{1}, y_{2}, \cdots, y_{k}>$, where $y_{j}(1 \leq j \leq k)$ is obtained from $j$-th elements of sequences assigned to the variables included in $B$. Let us denote the set of value-sequences for elements of $C l_{n}$ as $\left|C l_{n}\right|$. Because the sequences in question consist of $k$ elements, and the number of truth-values equals $m$, in total there is $m^{k}$ possible sequences. Therefore, there is a finite $n_{0} \leq m^{k}$, such that $\left|C L_{n_{0}}\right|$ contains no value-sequence which is not also the element of some $\left|C L_{n<n_{0}}\right|$.

Lemma 5. The value-sequence of any formula $B \in C L_{n>n_{0}}$ is identical to some element of $\left|C L_{n<n_{0}}\right|$.

Proof. Let $B \in C L_{n_{0}+1}$. By definition of $C L_{n_{0}+1}$, formula $B$ consists of the main connective, at least one formula from $C L_{n_{0}}$, 
and probably elements of $C L_{n_{i}<n_{0}}$. By definition of $n_{0}$, each valuesequence from $\left|C L_{n_{0}}\right|$ is also present in some $\left|C L_{n_{j}<n_{0}}\right|$. Therefore, by definition of $|C L|$, there is a set $\left|C L_{\max (i, j)+1}\right|$, which contains the value-sequence identical to $|B|$. Because $n_{i}<n_{0}$ and $n_{j}<n_{0}$, we have that $\max \left(n_{i}, n_{j}\right)+1 \leq n_{0},|B| \in\left|C L_{n \leq n_{0}}\right|$. From that, according to the definition of $n_{0}$, we obtain that $|B| \in\left|C L_{n<n_{0}}\right|$. The theorem is proved for $C L_{n_{0}+1}$. The generalization for $C L_{n>n_{0}}$ is obvious.

So the set $\left|C L_{1}\right| \cup\left|C L_{2}\right| \cup \cdots \cup\left|C L_{n_{0}}\right|$ contains all value-sequences possible in $\mathfrak{C}_{m}$ for formulas that contain no more than $m$ different variables. From this fact and Lemma 4 it follows that $\Gamma \vDash^{*}\left(\mathfrak{C}_{m}\right) B$ for each $\Gamma$ and $B$ iff $\Delta \vDash^{*}\left(\mathfrak{C}_{m}\right) E$ for every $\Delta$ and $E$ that consist exclusively of the elemnts of $C L_{1} \cup C L_{2} \cup \cdots \cup C L_{n_{0}}$.

This concludes the construction of the procedure for testing if $C(\mathfrak{A})=C(\mathfrak{B})$ for two arbitrary finite-valued matrices $\mathfrak{A}$ and $\mathfrak{B}$ for some propositional language $L$.

\section{References}

[1] KaLICKI, J., A test for the existence of tautologies according to manyvalued truth-tables, Journal of Symbolic Logic 15:182-184, 1950.

[2] Kalicki, J., A test for the equality of truth-tables, Journal of Symbolic Logic 17:161-163, 1952. 